\title{
Investigation of hydrostatic fluid forces in varying clearance turbomachinery seals
}

\author{
S.M.Tibos \\ GE Power \\ Newbold Road \\ CV21 2NH \\ United Kingdom \\ C.Georgakis \\ GE Power \\ Newbold Road \\ CV21 2NH \\ United Kingdom
}

\author{
J.A.Teixeira \\ Cranfield University \\ MK43 OAL \\ United Kingdom
}

\author{
S.Hogg \\ Durham University \\ Stockton Road \\ DH1 3LE \\ United Kingdom
}

\begin{abstract}
Varying clearance, rotor-following seals are a key technology for meeting the demands of increased machine flexibility for conventional power units. These seals follow the rotor through hydrodynamic or hydrostatic mechanisms. Forward facing step (FFS) and Rayleigh step designs are known to produce positive fluid stiffness. However, there is very limited modelling or experimental data available on the hydrostatic fluid forces generated from either design. A quasi-1D method has been developed to describe both designs and validated using test data. Tests have shown that the FFS and the Rayleigh step design are both capable of producing positive film stiffness and there is little difference in hydrostatic force generation between the two designs. This means any additional hydrodynamic features in the Rayleigh step design should have a limited effect on hydrostatic fluid stiffness. The analytical model is capable of modelling both the inertial fluid forces as well as the viscous fluid losses and the predictions are in good agreement with the test data.
\end{abstract}

\section{Nomenclature}

$\bar{B} \quad$ Dimensionless axial step position
$\bar{F}$

$\bar{H}$

$\bar{K}$

$\bar{P}$

$\bar{W}$

$\bar{X}$

$\dot{m}$

$\varepsilon$

$\gamma$

$f$

$\mu$

b

$D_{h}$

F

$g_{w}$

$h_{1}$

$h_{2}$

$i$

$L$

$M$

$P_{s}$

$P_{t}$
Dimensionless force

Dimensionless clearance

Dimensionless stiffness

Dimensionless pressure

Dimensionless circumferential step width

Dimensionless axial position

Mass flow rate $(\mathrm{kg} / \mathrm{s})$

Roughness coefficient

Specific heat ratio $\left(C_{p} / C_{v}\right)$

Friction factor (Darcy)

Viscosity $(\mathrm{Pa}-\mathrm{sec})$

Inlet axial groove length $(m)$

Hydraulic diameter $(m)$

Seal force $(N)$

Circumferential groove width $(m)$

Inlet gap height $(\mathrm{m})$

Seal gap $(m)$

Axial division

Axial seal length $(m)$

Mach number

Static pressure $(\mathrm{Pa})$

Total pressure $(\mathrm{Pa})$ 
$P_{\text {in }} \quad$ Upstream inlet pressure $(\mathrm{Pa})$

$P_{\text {out }} \quad$ Downstream outlet pressure $(\mathrm{Pa})$

$P_{t_{\text {loss }}} \quad$ Total pressure loss $(\mathrm{Pa})$

$R \quad$ Specific gas constant $(\mathrm{J} / \mathrm{K} / \mathrm{mol})$

$R e \quad$ Reynolds number

$T_{t} \quad$ Total temperature $(K)$

$W \quad$ Total circumferential seal width $(m)$

$x \quad$ Axial position $(m)$

\section{Introduction}

The need for compliant, varying clearance turbomachinery seals is driven by two main factors. Firstly, the current and future trends in flexible turbine operation for conventional power plants due to increased use of renewable technologies as noted by Messenger [1] and secondly, the cost effective higher efficiency operation available from improved seals well documented in several works such as that by Steinetz [2]. Varying clearance, rotor following seals can be designed to work through hydrodynamic or hydrostatic mechanisms or a combination of both as reported by Munson [3]. Hydrostatic load support alone is believed to be insufficient to offer the magnitude of fluid stiffness required to allow effective rotor following and stop seal to rotor contact, Galimutti [4]. This is particularly evident at very small clearances $(<50 \mu m)$. Contact of the seal with the rotor at high diameter and therefore high surface speed is detrimental to both performance but ultimately component life. Therefore, to avoid a rubbing event the seal must reliably exhibit rotor following behaviour. Hydrodynamic seals offer far higher levels of fluid stiffness, however these high stiffness values are only available at very small clearances $(<50 \mu m)$.

Effects such as rotor misalignment, thermal distortion and manufacturing tolerances mean that designing a seal to work at these clearances whilst avoiding contact becomes challenging. One solution is to use a hybrid of hydrodynamic and hydrostatic fluid force generation whereby the design clearance would be set on the verge of where hydrodynamic fluid forces are beginning to become effective and hydrostatic fluid forces are still partially effective. In this way the hydrostatic forces could be used as the main driver for movement of the seal whilst the hydrodynamic forces act as a buffer to stop contact during any rotor transient events.

One hydrostatic feature known to generate positive fluid stiffness is the forward facing step (FFS). This particular feature is utilised in the FRPALS (Film-riding pressure activated leaf seal) technology as described by Grondahl [5]. The design uses a double forward facing step where a larger step is used upstream and a smaller step used downstream. A similar FFS design with varying groove width was also added to the padded finger seal design by Zhang [6]. The first hydrostatic concept used by the HALO (hydrostatic advanced low leakage) seal technology was also a FFS design. This design was presented at the 2006 NASA Seal/Secondary Air System Workshop, Steinetz [7]. However, there is little experimental data or modelling of hydrostatic fluid forces on turbomachinery seals reported in literature.

Cheng et al.[8] were the first to model the FFS design on seals. The authors assumed negligible inertial fluid forces. However, at higher clearances Reynolds numbers increase and this assumption becomes questionable. Others, such as Zhang [6] have performed CFD analysis but only in the context of understanding flows through individual padded finger seals.

The Rayleigh step design is a progression of the FFS where three dimensional features are present that create a design that has both hydrodynamic and hydrostatic load support mechanisms. The downstream land creates the flow blockage necessary to mimic the FFS and provide hydrostatic load support whilst the axial groove edges, aided by the feed groove, create the hydrodynamic step bearing effect. The FFS and Rayleigh step designs can be seen in Figure 1 and Figure 2 respectively. The Rayleigh step design has been widely analysed from the perspective of pure hydrodynamic force generation e.g. DiRusso [9]. However, there is no modelling available on the isolated hydrostatic forces generated from the design. There is also no analysis of hydrostatic forces compared to an equivalent FFS.

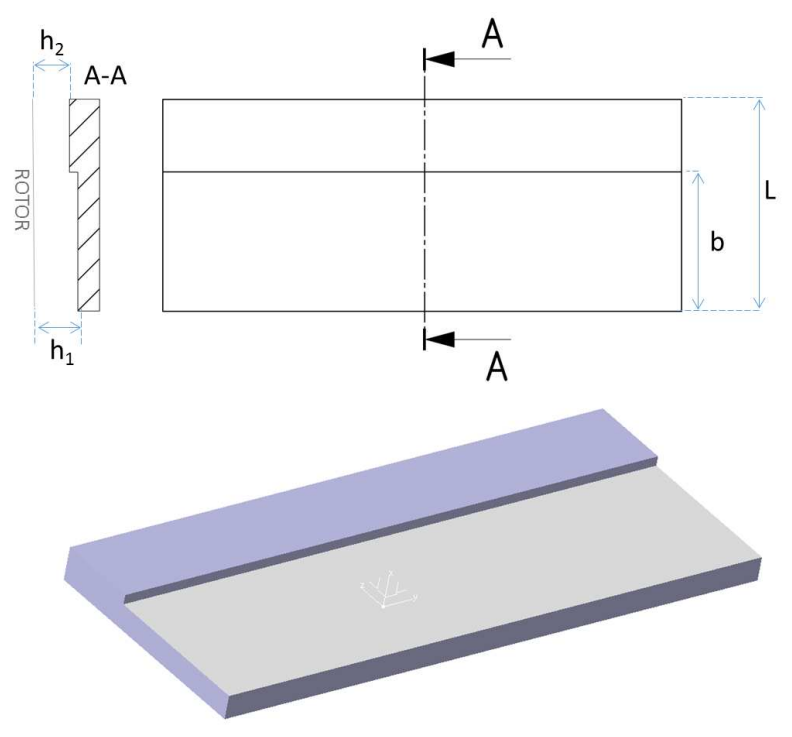

Fig. 1. Forward facing step design

Commercial CFD solvers could be used for investigating such designs. However, due to the small clearances and relatively large dimensions in the other two orthogonal flow directions the cell count needs to be very large in order to limit the number of high aspect ratio cells and maintain computational results accuracy. This is particularly challenging for the Rayleigh step design where the geometry can not be simplified to an axis-symmetric or 2D planar domain like the FFS. Therefore, a need exists for a lower fidelity quasi-1D or 2D model to analyse such designs. Satisfactory simulation of these complex flows using such a strategy also opens up opportunities for rapid analysis that can be used as part of optimisation or aid further design enhancements. 


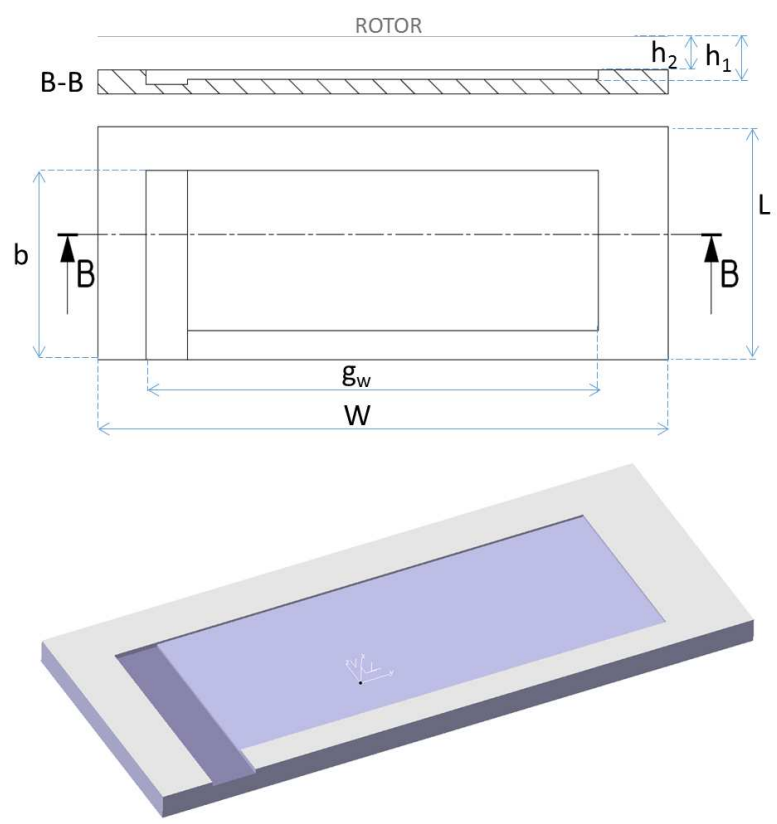

Fig. 2. Rayleigh step design

\section{Analytical model}

\subsection{Model description}

The analytical modelling of Cheng [8] can be improved by considering the inertial effects of the fluid. To accomplish this the fluid can be modelled as a quasi-1D compressible flow. A total pressure drop through the seal gap will exist due to frictional viscous losses so the flow cannot be considered isentropic. This drop in total pressure is greater through the smaller seal gap. These losses can be explained by frictional losses synonymous with pipe flow frictional losses whereby the loss in total pressure can be described by Eq.(1).

$$
P_{t_{\text {loss }}}=\frac{f \gamma P_{t} M^{2} L}{2 D_{h}}
$$

In order to solve the fluid field an initial guess of the unit mass flow is needed. This can be done by first considering isentropic conditions, using the smallest area and upstream and downstream pressure conditions as shown in Eq.(2) which is in the same form as presented by Herrmann [10].

$$
\dot{m}=\frac{P_{t} h_{2}}{\sqrt{T_{t}}} \sqrt{\frac{2 \gamma}{R(\gamma-1)}\left[\left(\frac{P_{\text {in }}}{P_{\text {out }}}\right)^{\frac{2}{\gamma}}-\left(\frac{P_{\text {in }}}{P_{\text {out }}}\right)^{\frac{\gamma+1}{\gamma}}\right]}
$$

The algorithm used to solve for pressure distribution and mass flow through the seal can be seen in Figure 3.

The algorithm in Figure 3 works by first estimating a mass flow based on upstream and downstream pressure conditions. A fraction (default is $\frac{1}{2}$ ) of this mass flow is used as the starting point.

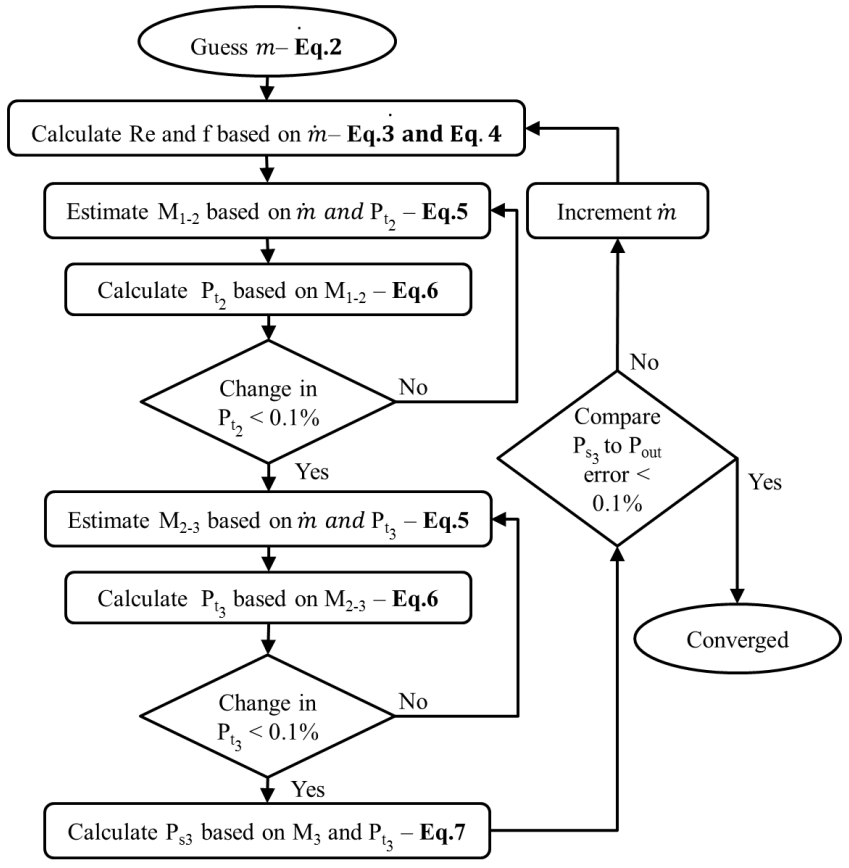

Fig. 3. Algorithm used for solving pressures, Mach number and mass flow quantities

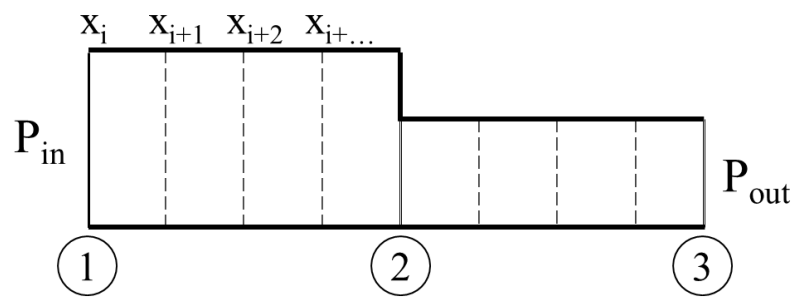

Fig. 4. Computational domain showing stations and divisions

The Reynolds number is calculated using Eq.(3). This value is then used in the Colebrook-White equation, Eq.(4) where the hydraulic diameter for a long (2D) gap is found using $D_{h}=2 h_{2}$. The calculation assumes a unit depth where the area term is replaced by a length, in this case the gap height $h_{2}$. An estimate of friction factor (Darcy-Weisbach) is then made using Eq.(4. The surface roughness is omitted.

$$
R e=\frac{\dot{m} D_{h}}{h_{2} \mu}
$$

$$
\frac{1}{\sqrt{f}}=-2 \log \left(\frac{\varepsilon}{3.7 D_{h}}+\frac{2.51}{\operatorname{Re} \sqrt{f}}\right)
$$

The space between stations 1 to 2 (as seen in Figure 4) is discretised using a user-defined number of divisions. The algorithm iterates to find the Mach number and total pressure at each of these divisions. This is achieved by using Eq.(5) to 
guess and iterate to find Mach number and then solve Eq.(6) to find the total pressure at each division. This part of the algorithm converges when the exit total pressure no longer changes beyond a set target (default $=0.1 \%$ ).

$$
\begin{gathered}
\dot{m}=\frac{P_{t_{i}} h_{i}}{\sqrt{R T_{t}}} \frac{\sqrt{\gamma} M_{i}}{\left(1+\frac{\gamma+1}{2} M_{i}^{2}\right)^{\frac{\gamma+1}{2(\gamma-1)}}} \\
P_{t_{i}}=P_{t_{i-1}}-\frac{P_{t_{i-1}}\left(x_{i}-x_{i-1}\right) f M_{i}^{2}}{2 h_{i}}
\end{gathered}
$$

The conditions found at station 2 are then used as input to find Mach number and total pressure at station 3 using this same technique. These conditions are then used to estimate an outlet static pressure using Eq.(7).

$$
P_{s_{3}}=P_{t_{3}}\left(1+\left(\frac{\gamma-1}{2}\right) M_{3}^{2}\right)^{\left(\frac{-\gamma}{\gamma-1}\right)}
$$

The guess of static pressure from Eq.(7) is compared to the real boundary condition. Once the difference between these two pressures reaches a target value (e.g. $0.1 \%$ ) the solution is converged. The mass flow is gradually varied on each loop until this convergence criteria is reached. It should be noted that this model works equally well for choked flow with some modifications to the method. However, the pressure ratios considered in this study are small and will only produce subsonic flow therefore this addition to the method is omitted.

\subsection{Application to Rayleigh step design}

The quasi-1D tool is extended to solve multiple axial flow paths and the results averaged to give an estimate for a $2 \mathrm{D}$ seal pad area. The set-up of this model can be seen in Figure 5. The model solves for 1) flow through the feed groove where the step height is the feed groove depth 2) the flow in the main groove zone where the upstream land is neglected and the step height is input as the main groove depth 3) flow across the main land area where there is no step encountered. The modelled profiles can be seen in the bottom of Figure 5 .

The force is then calculated by multiplying the 1D unit forces along each axial flow line by the relative circumferential length. For example, in the case of line 2 (main groove) the unit force would be multiplied by the circumferential groove length.

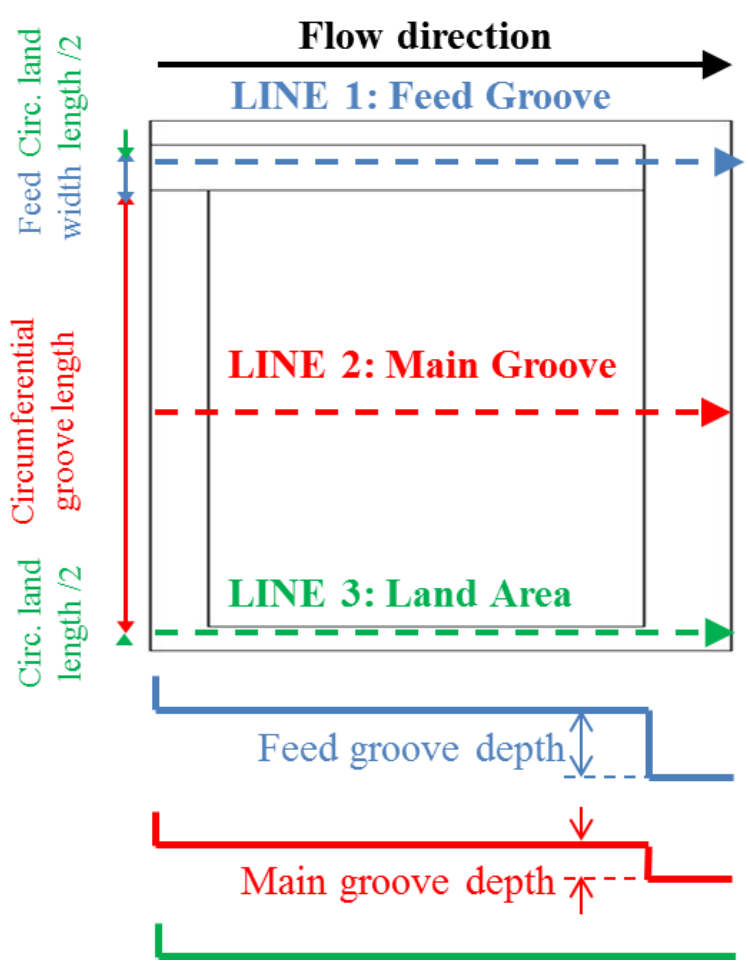

Fig. 5. Set-up of 1D tool for the Rayleigh step design

\section{Experimental work \\ 3.1 Test rig design}

A schematic representation of the test rig at Durham University is shown in Figure 6. The test rig is an oblong pressure vessel, constructed from three millimetre thick steel plate, with a flange at inlet and an exit to atmosphere. There is a central dividing wall to separate the upstream and downstream sides of the seal geometry. The seal test pieces slot into an aluminium extrusion allowing the seal to be quickly swapped between tests. The aluminium slot section was suspended from a load cell. This allowed the difference in force produced in the vertical direction by the upstream pressure on top of the seal and the pressure generated by the seal geometry underneath it to be measured. The load cell was fixed rigidly to a leveling bar. This bar had the load cell fixed in a central position, and a bolt at each end, which were screwed through the lid of the box. This arrangement meant that any cross-flow roll of the seal geometry could be eliminated by screwing up or down one of the sides to maintain the specified clearance across the width of the seal. Small gaps (less than $0.1 \mathrm{~mm}$ ) were designed between the central dividing wall and the side walls. These gaps were essential so that there was no interference of the force measurement being measured by the load cell. However, these gaps create additional leakage paths for the flow. Several preliminary test runs were made at various primary gap heights to ensure that the results were unaffected by the side wall gaps.

The central dividing wall separates the high and low pressure sides of the seal arrangement allowing a pressure differential between the two sides to be created. This meant that a large force was produced forcing the whole seal assem- 


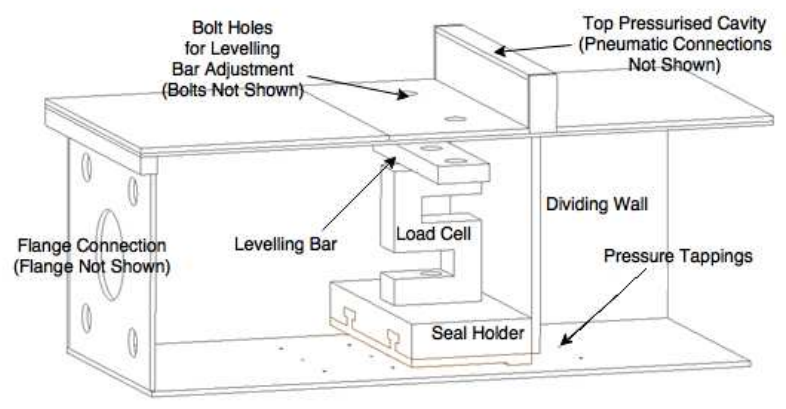

Fig. 6. Rig Schematic

bly towards the outlet of the rig. The load cell and leveling bar were not designed to withstand this direction of lateral loading and the rotation this induced would have introduced error in the results. To counteract this, a threaded hole was drilled into the front of the aluminium slot piece, and a thin threaded bar was taken from this part and bolted to the front of the rig stopping the whole assembly being pushed backwards. This solution also had the benefit of being adjustable by moving the bolts along the bar, therefore making the rig able to compensate for any front to back pitch of the seal in the rig.

Beneath the horizontal centreline of the seal geometry, on the base of the rig, there are a series of eight closely spaced pressure tappings ( $\mathrm{C} 1$ to $\mathrm{C} 8)$ as shown in Figure 7. These holes are positioned so that a detailed assessment of the static pressure profile over the length of the seal could be measured.

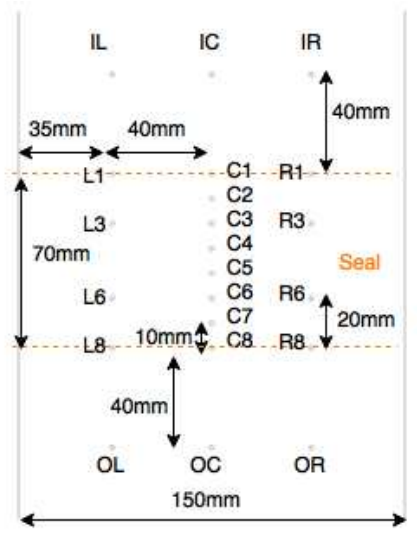

Fig. 7. Pressure tappings location

\subsection{Testing method}

The blow-down facility at Durham University was used to carry out the testing. This facility allows for a large volume of air to be pressurised and stored, and then released at a constant pressure by varying the position of the outlet valve as the tank empties. This arrangement is shown schematically in Figure 8.

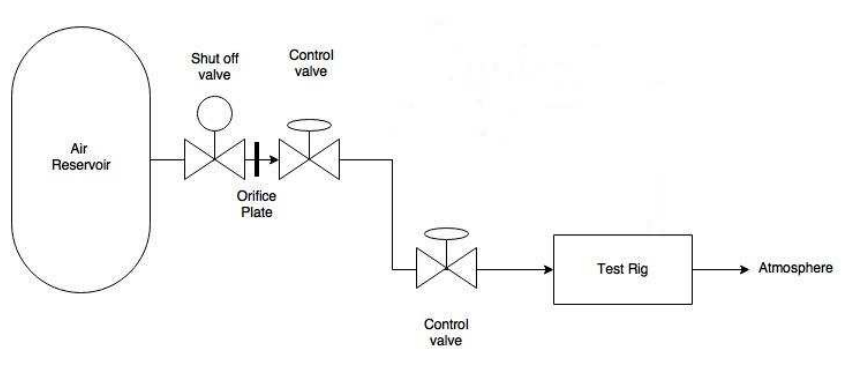

Fig. 8. Blowdown facility schematic

The key dimensional parameters and boundary conditions are detailed in Table 1. The seal designs were tested over a range of upstream to downstream pressure differentials, from 0.10 bar to 0.40 bar. The exit pressure was always atmospheric pressure.

Table 1. Key dimensional parameters and boundary conditions

\begin{tabular}{lll}
\hline Parameter & Value & Units \\
\hline Step height & $0.6-0.7$ & $\mathrm{~mm}$ \\
Clearance & $0.1-0.75$ & $\mathrm{~mm}$ \\
$\bar{H}$ & $0.14-1.25$ & - \\
Seal length & 70 & $\mathrm{~mm}$ \\
Seal width & 150 & $\mathrm{~mm}$ \\
Inlet gauge pressure & $0.1-0.40$ & $\mathrm{bar}$ \\
Outlet gauge pressure & 0 & bar \\
\hline
\end{tabular}

The central dividing wall was part of the seal-load cell assembly, and therefore moved vertically as the seal was moved to set the clearance. The clearance was varied from 0.14 to 1.25 times the seal step height. The results from this series of experiments were then used to plot dimensionless force and dimensionless pressure curves.

\subsection{Experimental Results}

The results are presented in terms of non-dimensional force $(\bar{F})$, non-dimensional clearance $(\bar{H})$, non-dimensional pressure $(\bar{P})$ and non-dimensional axial position $(\bar{X})$. The use of non-dimensional values is important, as the geometries tested were not the same size as would be implemented inside a real machine. Different size seals of the same design may be needed in different turbines, for example in a high pressure turbine compared to a low pressure turbine. The non-dimensional values are calculated as shown in $\mathrm{Eq}(8,9$, 10 and 11).

$$
\bar{H}=\frac{h_{2}}{h_{1}-h_{2}}
$$




$$
\begin{gathered}
\bar{F}=\frac{F}{\left(P_{\text {in }}-P_{\text {out }}\right) A} \\
\bar{P}=\frac{P-P_{\text {out }}}{P_{\text {in }}-P_{\text {out }}} \\
\bar{X}=\frac{x_{i}}{L}
\end{gathered}
$$

The experimental results showing dimensionless force and pressure in the axial direction for both the Rayleigh step and the FFS can be seen in Figures 9 and Figure 10 respectively.

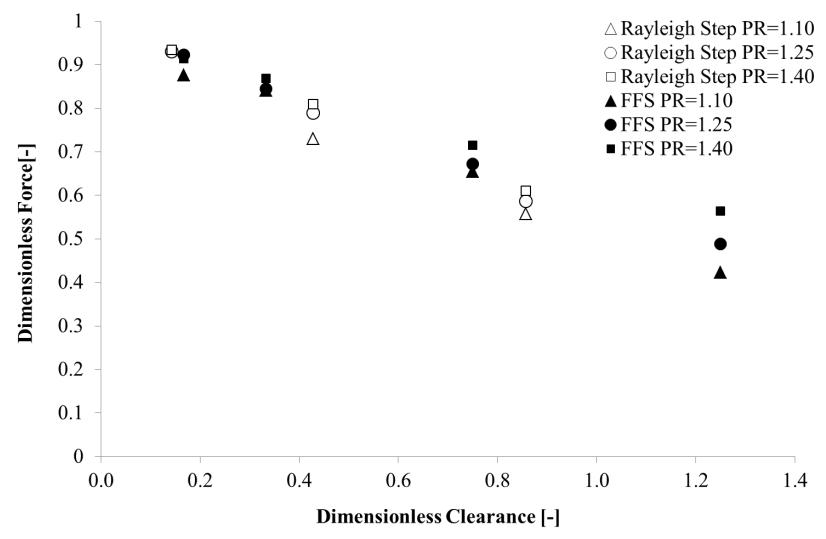

Fig. 9. Experimental results of dimensionless force against dimensionless clearance for Rayleigh step and FFS designs

The plots of dimensionless force for the Rayleigh step and FFS shown in Figure 9 are very similar. There appears to be a small reduction in dimensionless force compared to the simple FFS. However, the slope of the lines are similar showing little or no reduction in fluid stiffness.

The lines of dimensionless pressure in the axial direction for the Rayleigh step and FFS shown in Figure 10 are also very similar. The main difference between the two sets of experimental data occurs at inlet to the seal at the first pressure tapping. At this location the gap in the Rayleigh step design is smaller than the FFS. The flow velocity has to therefore increase which results in a drop in local static pressure at this location. This reduced static pressure means a smaller resultant force which explains the small differences between the dimensionless force plots in Figure 9.

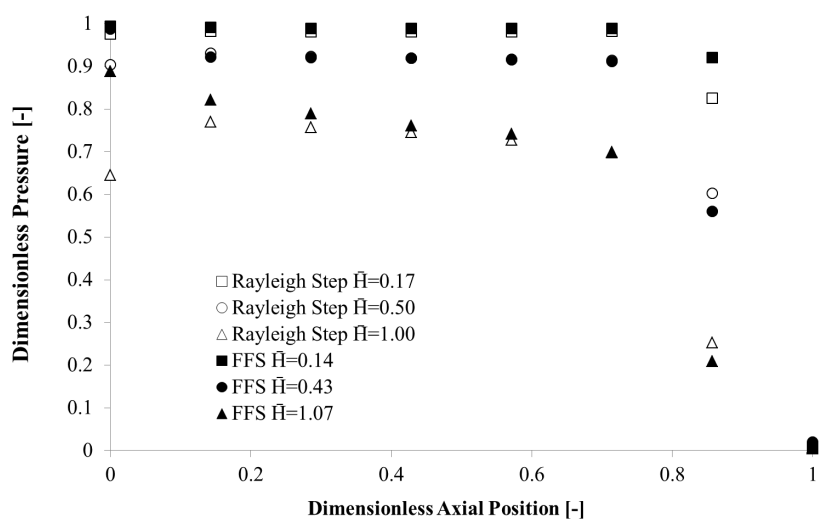

Fig. 10. Experimental results of dimensionless pressure against dimensionless axial position for Rayleigh step and FFS designs at a pressure ratio of 1.4

\section{Comparison of experimental and analytical data}

The experimental results are compared to the model of Cheng [8] (where applicable) and the analytical model developed in this study (as described in section 2).

Firstly, the FFS is compared. Plots of dimensionless pressure in the axial direction and dimensionless force against dimensionless clearance at a pressure ratio of 1.4 can be seen in Figure 11 and Figure 12 respectively.

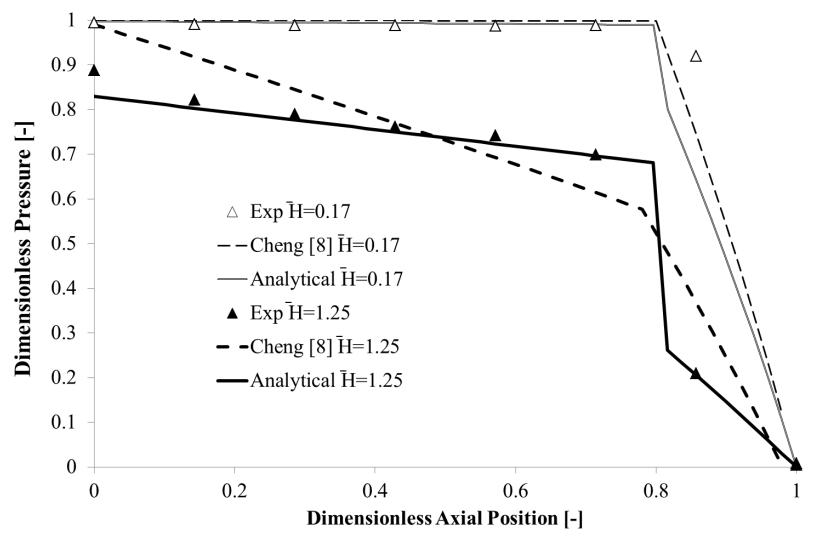

Fig. 11. Results comparison of dimensionless pressure against dimensionless axial direction between experiments and analytical models for the FFS design at a pressure ratio of 1.4

In Figure 11 the comparison at $\bar{H}=0.17$ shows that both analytical models are in reasonable agreement with the test data. At this small clearance case the assumptions of viscous dominated flow used by Cheng [8] is justified. The analytical model (described in section 2) also handles this case well, the small clearance creates relatively high frictional losses which results in static pressure being close to total pressure through the seal gap. For the clearance case of $\bar{H}=1.25$ the assumptions in the model of Cheng begin become questionable. At this clearance the described analytical model 


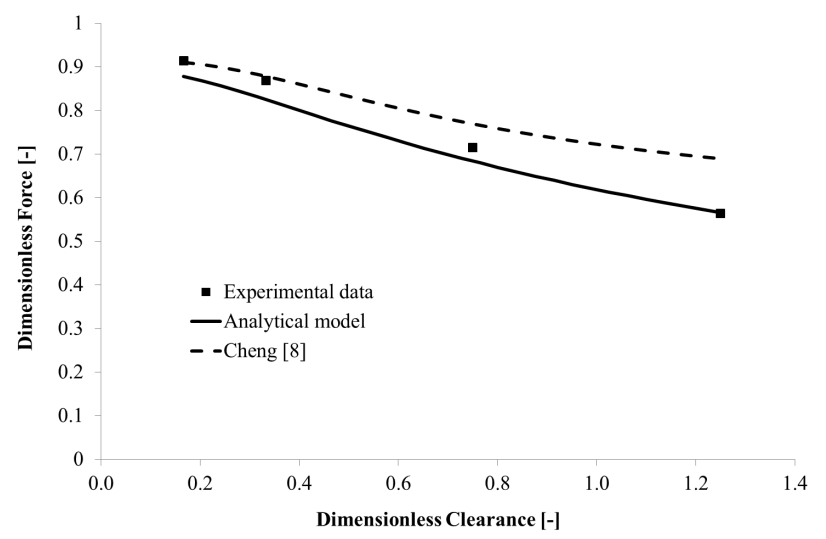

Fig. 12. Results comparison of dimensionless force against dimensionless clearance between experiments and analytical models for the FFS design at a pressure ratio of 1.4

matches the test data closer across a wider range of applicable clearances. The static pressure drop at inlet to the seal is well captured as well as the pressure drop at the step region. The location of the pressure tappings in the test rig means that the detailed pressure profile around the step region is not captured experimentally. However, the points that were recorded show good agreement (within 5\%).

The plot of dimensionless force in Figure 12 also shows a better matching with experimental data using the model described herein (within 4\%) compared to the model of Cheng [8]. Again, this is most evident for cases where the clearances are larger.

The analytical model is also compared to the experimental data for the Rayleigh step design. In this comparison the axial pressure drop through the main groove section is recorded experimentally. The plot of dimensionless pressure in the axial direction at a pressure ratio of 1.4 can be seen in Figure 13

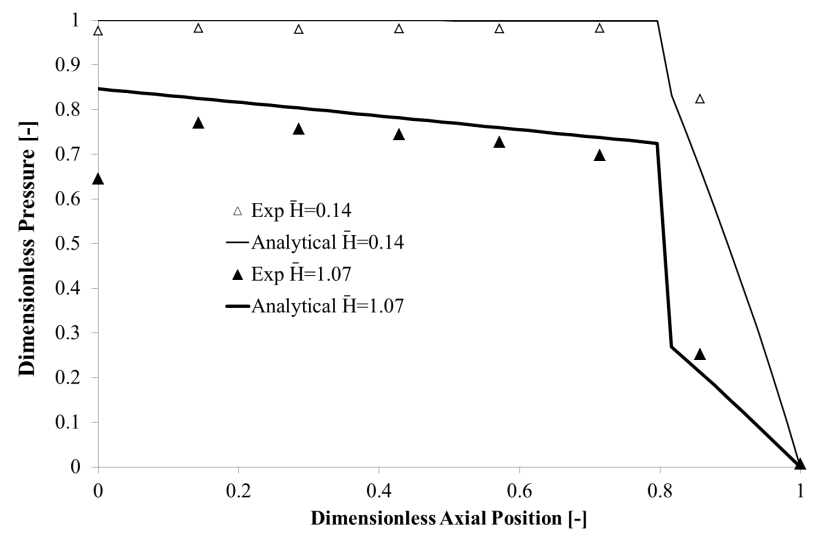

Fig. 13. Results comparison of dimensionless pressure against dimensionless axial direction between experiments and analytical models for the Rayleigh step design at a pressure ratio of 1.4

Both the cases of $\bar{H}=0.14$ and $\bar{H}=1.07$ demonstrate a reasonable agreement between the experimental data and the analytical model. The pressure drop across the step region is well captured, however, the initial pressure drop due to the upstream land region on the Rayleigh step is not captured. This design feature is not included in the model for simplicity and the effect is therefore not expected to be replicated. Nevertheless, the total pressure loss before and after the step are modelled sufficiently which is key to accurately predicting the fluid forces on the seal pad. Calculations of dimensionless pressure are within 5\% of the test data (apart from on entry to seal pad).

A comparison of the dimensionless force is also made for the 1.4 pressure ratio case. The plot of dimensionless force against dimensionless clearance for the Rayleigh step design can be seen in Figure 14.

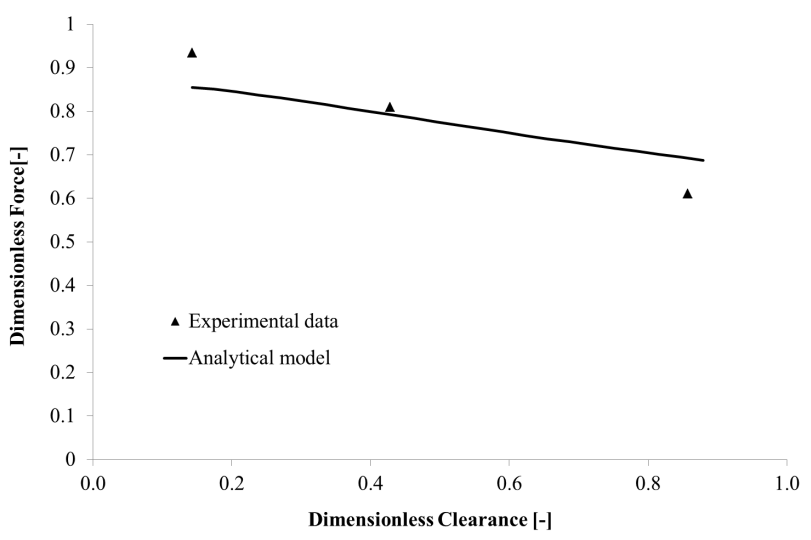

Fig. 14. Results comparison of dimensionless force against dimensionless clearance between experiments and analytical models for the Rayleigh step design at a pressure ratio of 1.4

The plot in Figure 14 shows a reasonable agreement with the test data, the maximum difference between data points is within $13 \%$. However, it should be noted that the dimensionless force is slightly under predicted at low clearance and over predicted at high clearance.

\section{Effects of land to groove length ratios}

The extended quasi-1D tool for Rayleigh step designs is used to explore the impact of varying the amount of land to groove width ratio in the circumferential direction and varying the axial position of the step (varying the ratio of groove to land in the axial direction). The axial step position is defined by $\bar{B}$ as shown in Eq.(12) and the circumferential width is defined by $\bar{W}$ as show in Eq.(13).

$$
\bar{B}=\frac{b}{L}
$$

$$
\bar{W}=\frac{g_{w}}{W}
$$


Three different step positions are assessed $\bar{B}=0.4, \bar{B}=$ 0.6 and $\bar{B}=0.8$. Three different circumferential land to groove ratios were also assessed $\bar{W}=0.33, \bar{W}=0.63$ and $\bar{W}=0.93$. The dimensionless stiffness shown in Eq.(14) was used for this assessment.

$$
\bar{K}=\frac{d F}{d h_{2}} \frac{h_{2}}{\left(P_{\text {in }}-P_{\text {out }}\right) A}
$$

The results for dimensionless stiffness against dimensionless clearance with varying land to groove width ratio in the circumferential direction and varying axial position of the step can be seen in Figure 15 and Figure 16 respectively.

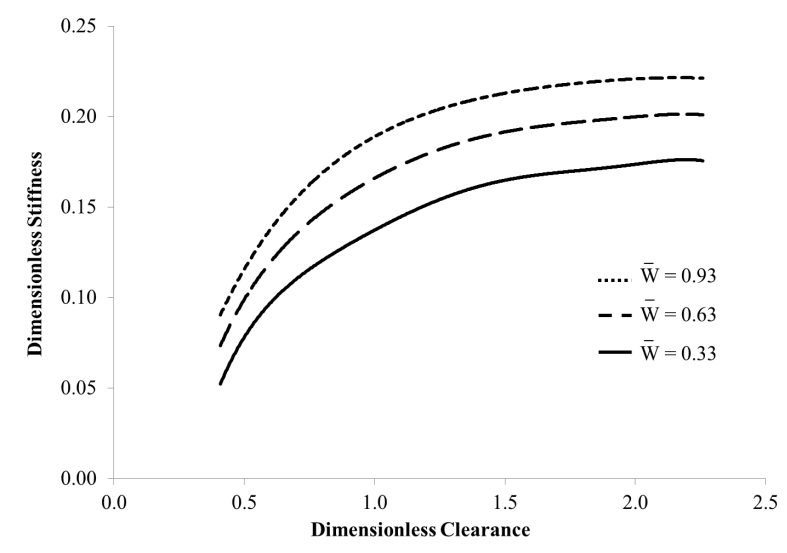

Fig. 15. Comparison of dimensionless stiffness against dimensionless clearance for varying land to groove width ratio in the circumferential direction (Rayleigh step design)

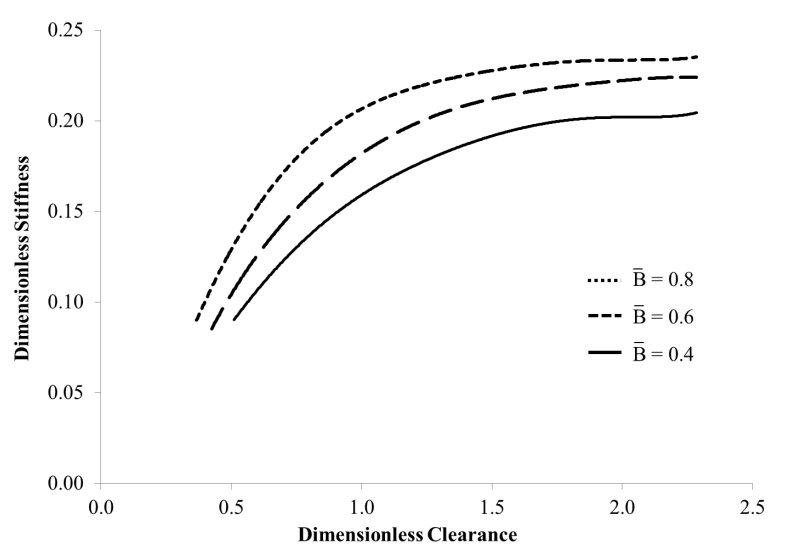

Fig. 16. Comparison of dimensionless stiffness against dimensionless clearance for varying axial position of the step (Rayleigh step design)

The curves in both Figure 15 and Figure 16 show that for smaller values of $\bar{B}$ and $\bar{W}$ there is a reduction in dimensionless stiffness. This means that there is a lower hydrostatic fluid stiffness when the step is further forward in the axial position, i.e. there is an increased land area in proportion to the overall area. The fluid stiffness is also reduced when the main groove takes up a smaller proportion of the total circumferential length of the seal pad.

In both plots there is a reduction of fluid stiffness at lower dimensionless clearance values and a sustained fluid stiffness at high dimensionless clearances. This trend is seen for all land to groove ratios.

\section{Discussion}

Both the FFS and the Rayleigh step design produce increasing force with reduced clearance. As a result both designs could be used for a hydrostatic film riding seal design. The FFS is currently being used by the FRPALS technology with successful results from initial static tests [11]. Other similar film-riding seal designs could work equally well by using this step feature. However, whether sufficient fluid stiffness is able to be generated at minimum clearance using only the hydrostatic load support from a FFS is questionable.

In the two plots of dimensionless stiffness (Figure 15 and Figure 16) there is a reduction of stiffness at lower dimensionless clearances. This reduction in fluid stiffness is consistent with the argument that using hydrostatic fluid forces alone would not generate sufficient load support particularly at low clearance. To combat this a hybrid design that utilises hydrodynamic forces at low clearance could be useful. Further work would be required to understand the combination of hydrostatic and hydrodynamic fluid forces across the range of operating clearances that ensures optimal performance can be achieved.

The test results also showed that there is little difference between the two designs (FFS and Rayleigh step) and that the additional hydrodynamic features in the Rayleigh step design should have a limited effect on the hydrostatic fluid stiffness but may marginally lower the fluid forces equally across the range of clearances. This result is partly expected as the designs are similar. The difference lies in the additional three dimensional features present in the Rayleigh step design. Hence, what has been shown is that the three dimensional features necessary for hydrodynamic stiffness at low clearance are not greatly impeding the function of the axial step feature that generates hydrostatic stiffness at higher relative clearance. This result means that the Rayleigh step design could indeed be used as a hybrid load support design capable of producing both hydrodynamic and hydrostatic fluid forces in a manner that limits the risk of seal and rotor contact. It should be noted that once rotation is included the Rayleigh step design will produce significantly more lift and stiffness from the hydrodynamic features whereas the FFS will produce no force from this mechanism.

The experimental results have also been compared to a quasi-1D analytical model described herein as well as the model of Cheng [8]. The described model shows better agreement with the test data particularly at larger seal gaps. The model is capable of capturing both the inertial fluid forces as well as the viscous fluid losses due to the friction on 
the seal and rotor walls. This makes the model more applicable across a range of operating conditions and clearances. This capability is lacking in the model of Cheng which is formulated on the basis of the Reynolds equation where viscous forces are presumed to dominate and inertial forces are neglected. The inclusion of inertial fluid forces is also useful for getting accurate predictions of the flow at off-design conditions where the seal gaps may be higher than the design clearances. This is particularity useful for determining the resultant force at machine start-up to ensure the seal begins to close the radial gap and move into the desired running position.

The analytical model is also able to predict the fluid forces on the 3D design of the Rayleigh step geometry using three individual quasi-1D axial lines. This capability speeds up the analysis of the geometry which will enable designs to be quickly optimised and checked for performance robustness. The extended quasi-1D model was also used to investigate the effect of varying the amount of land to groove width ratio in the circumferential direction and varying the axial position of the step. The results showed that for smaller values of $\bar{B}$ and $\bar{W}$ there is a reduction in dimensionless stiffness. This shows that in order to maximise the fluid stiffness from hydrostatic forces the land areas in both the axial and circumferential directions should be as small as possible. This result is not surprising, as the groove to land ratios approach their maximum value of 1 the design then tends towards that of a simple FFS. However, by minimising land area this would likely have an effect on the generation of hydrodynamic forces. There should therefore be a consideration of the optimal design for both fluid force generation mechanisms.

By modelling the Rayleigh step using the three line quasi-1D method the interaction between the three flow paths are ignored. The differences in the geometry in these three locations will no doubt create discordant axial pressure profiles which will result in pressure gradients in the perpendicular flow direction driving some amount of cross flow. The inclusion of the rotating wall in a real application will also change the flow field and the associated moving wall boundary condition should therefore be added to the method. The hydrostatic analysis of the Rayleigh step design could then be coupled with a hydrodynamic analysis using a Reynolds equation method to give a more complete assessment of both hydrostatic and hydrodynamic fluid forces.

\section{Conclusions}

This paper describes a quasi-1D method capable of assessing the hydrostatic fluid forces for a FFS (forward facing step) and Rayleigh step design. The validation of the developed analytical method is done using experimental data. The main findings of the work can be summarised below.

The experimental results have shown that the FFS and the Rayleigh step design are both capable of producing positive film stiffness i.e. increasing force with reduced clearance. Both designs produce similar hydrostatic fluid force and pressure results. There is some reduction of fluid force and stiffness in the Rayleigh step design particular when the groove to land ratio is reduced, however, this reduction is minimal.

The analytical model is in good agreement with the test data for the FFS design. The model is capable of capturing both the inertial fluid forces as well as the viscous fluid losses due to the friction on the seal and rotor walls. As a consequence the described model produces a more accurate result compared to what has been reported in literature by Cheng [8], particularly at larger relative clearances. Calculations of pressure were within $5 \%$ of the test data and calculations of force were within $4 \%$.

The analytical model is able to adequately predict the fluid forces on the three dimensional design of the Rayleigh step geometry. This is achieved by using three individual quasi-1D axial lines. Calculations of pressure were also within $5 \%$ of the test data, apart from on entry to seal pad. Whereas, calculations of force deviated more from the test data but were within $13 \%$. To maximise the hydrostatic fluid stiffness in a Rayleigh step design the land areas in both the axial and circumferential directions should be minimised to more closely resemble the FFS.

\section{Acknowledgements}

The authors would like to acknowledge the contributions of Matthew Mundy in the design and build of the experimental rig at Durham University and the efforts of Andrew Messenger in supporting the experimental test runs.

\section{References}

[1] Messenger, A., Williams, R., Ingram, G., Hogg, S., Tibos, S., and Seaton, J., 2015. "A dynamic clearance seal for steam turbine application”. ASME Paper No. GT2015-43471.

[2] Steinetz, B., Hendricks, R., and Munson, J., 1998. Advanced seal technology role in meeting next generation turbine engine goals. Tech. Rep. 1998-206961 AVTPPS Paper No. 11, NASA.

[3] Munson, J., 1993. "Testing of a high performance compressor discharge seal”. Paper No. AIAA 93-1997.

[4] Galimutti, P., Sawicki, J., and Fleming, D., 2009. "Analysis of finger seal lift pads". ASME Paper No. GT2009-59842.

[5] Grondahl, C. M., and Dudley, J. C., 2010. "Film-riding leaf seals for improved shaft sealing". ASME Paper No. GT2010-23629.

[6] Zhang, H., Zheng, Q., Yue, G., and Deng, Q., 2012. "On the lifting performance of a finger seal with pad grooved”. ASME Paper No. GT2012-68855.

[7] Steinetz, Bruce, M., Hendricks, R. C., and Delgado, I., 2007. Nasa seal/secondary air system workshop. Tech. Rep. CP-2007-214995 VOL 1 E-16198-1, NASA.

[8] Cheng, H. S., Chow, C. Y., and Wilcock, D. F., 1968. "Behavior of hydrostatic and hydrodynamic non- 
contacting face seals". Journal of Lubrication Technology(April), pp. 510-519.

[9] DiRusso, E., 1982. Film thickness measurements for spiral groove and rayleigh step lift pad self-acting face seals. Tech. Rep. 2058, NASA.

[10] Herrmann, N., Dullenkopf, K., and Bauer, H., 2013. "Flexible seal strip design for advanced labyrinth seals in turbines". ASME Paper No. GT2013-95424.

[11] Kirk, T., Bowsher, A., Crudgington, P., Pawlak, A., Grondahl, C., and Dudley, J., 2016. "Film riding pressure activated leaf seal proof of concept". Paper No. AIAA 2016-4920. 